\title{
Editorial Message: Special Issue on Fuzzy Brain-Computer Interface Systems
}

\author{
Yi-Hung Liu ${ }^{1} \cdot$ Tzyy-Ping Jung ${ }^{2} \cdot$ Chin-Teng Lin ${ }^{3} \cdot$ Lun-De Liao ${ }^{4}$
}

Published online: 22 March 2017

(C) Taiwan Fuzzy Systems Association and Springer-Verlag Berlin Heidelberg 2017

Brain-computer interface (BCI) has become a key research topic in the fields of translational/cognitive neuroscience, neuroengineering, and neurorehabilitation. BCI systems can serve as an effective neurofeedback mechanism in various applications ranging from clinical assessment, health care, intelligent transportation, education, and robotic control to entertainment, and thus, a wide spectrum of users/patients can benefit from this novel technology.

The last decade has witnessed remarkable advances in BCI research. However, there still exist challenges that need to be overcome. For example, nearly all the current BCI studies were conducted in well-controlled laboratory settings. It remains unclear how well the existing BCI systems work when faced with real patients and implemented in unconstrained and dynamic environments. To address these issues caused by the uncertainties in realworld BCI applications, many researchers and groups have started to exploit fuzzy set theory and systems to the BCI research community and have shown increasing achievements.

The special issue on fuzzy brain-computer interface systems was called for papers during December 2015-July 2016. The objective of this special issue is to explore up-todate fuzzy set solutions for BCI systems and applications. The criteria for the review were based on technical contributions, novelty, and completeness. After the rigorous

Yi-Hung Liu

yhliu@ntut.edu.tw

National Taipei University of Technology, Taipei, Taiwan

2 University of California - San Diego, San Diego, CA, USA

3 National Chiao Tung University, Hsinchu, Taiwan

4 National Health Research Institutes, Miaoli, Taiwan review process, six papers were accepted for publication in this IJFS special issue. All the six papers successfully introduced fuzzy methods (e.g., fuzzy entropy, fuzzy neural network, fuzzy support vector machine) to address critical issues related to different BCI topics, including the segmentation of brain images, steady-state visually evoked potentials (SSVEP), P300 event-related potentials, EEG activities induced by motor imagery, and EEG signals induced by real exercise. These articles provide novel and useful results covering "P300-based Brain-Computer Interface with Latency Estimation Using ABC-based Interval Type-2 Fuzzy Logic System," "Improvement of Classification Accuracy in a Phase-Tagged Steady State Visual Evoked Potential-Based Brain-Computer Interface using Adaptive Neuron-Fuzzy Classifier," "A Single Channel SSVEP based BCI with a Fuzzy Feature Threshold Algorithm in a Maze Game," "EEG Classification of Imaginary Lower Limb Stepping Movements Based on Fuzzy Support Vector Machine with Kernel-Induced Membership Function," “Analysis of Electroencephalography Alteration During Sustained Cycling Exercise Using Power Spectrum and Fuzzy Entropy," and "Spatial Possibilistic Fuzzy C-Mean Segmentation Algorithm integrated with Brain Mid-Sagittal Surface Information."

This editorial message not only delineates the paper submission and rigorous review, but also assures the quality of the papers in this special issue of IJFS. As the guest editors of this special issue on fuzzy brain-computer interface systems, we appreciate the author's patience and effort for revising their submissions according to the reviewers' comments. 